\title{
Effects of copper sulphate and rumen-protected copper sulphate addition on growth performance, nutrient digestibility, rumen fermentation and hepatic gene expression in dairy bulls
}

KEY WORDS: copper, daily gain, microbiota, enzyme activity, protein metabolism, dairy bulls

Received: $\quad 2$ July 2020

Revised: 20 October 2020

Accepted: 30 November 2020

\author{
S. La, C. Wang, Q. Liu', G. Guo, W. Huo, J. Zhang and C. Pei \\ Shanxi Agricultural University, College of Animal Science, Department of Animal \\ Nutrition and Feed Science, Taigu, 030801, Shanxi, P.R. China
}

${ }^{1}$ Corresponding author:

e-mail: liuqiangabc@163.com

\begin{abstract}
In the study the effects of copper sulphate (CS) and rumenprotected copper sulphate (RPCS) addition on performance, nutrient digestibility, rumen fermentation and hepatic gene expression in bulls were evaluated. Thirtysix Holstein bulls were randomly assigned to three treatments: control (without Cu supplementation), CS (8 mg/kg dry matter (DM) Cu from CS) and RPCS (8 mg/kg DM Cu from RPCS). Dietary Cu addition did not affect DM intake and average daily gain, but increased apparent nutrients digestibility. Ruminal $\mathrm{pH}$, propionate percentage and ammonia- $\mathrm{N}$ concentration decreased, but total volatile fatty acids concentration and acetate percentage increased with dietary $\mathrm{Cu}$ inclusion. Activities of carboxymethyl-cellulase, xylanase and laccase and populations of total bacteria, Butyrivibrio fibrisolvens and Ruminococcus albus increased, but a-amylase activity decreased with dietary Cu provision. In bulls receiving RPCS supplementation greater activities of xylanase, pectinase and a-amylase and populations of Ruminococcus flavefaciens and Butyrivibrio fibrisolvens were noted than in those receiving CS addition. Activities of laccase and protease were lower in RPCS group than in CS one. Liver Cu concentration was the highest in RPCS animals, followed by CS, and then control ones. Hepatic expressions of insulin-like growth factor-1 (IGF-1), IGF-1 receptor, phosphoinositide 3-kinase and ribosomal protein $S 6$ kinase were reduced by RPCS, but were not affected by CS addition. Hepatic expression of mammalian target of rapamycin was the lowest in RPCS group, followed by CS, and then control ones. It is suggested that dietary $\mathrm{Cu}$ addition promoted nutrients digestion and ruminal fermentation, and replacement of CS with RPCS down-regulated hepatic protein synthesis metabolism genes expression.
\end{abstract}

\section{Introduction}

Copper $(\mathrm{Cu})$ is an essential trace element, and is mainly used as a cofactor of redoxactive enzyme in ruminants (NRC, 2001). It was found that dietary copper sulphate (CS) addition increased feed efficiency in calves (Gengelbach and Spears, 1998) and unsaturated fatty acid content of longissimus dorsi muscle in steers (Engle and Spears, 2000a). In other studies it was reported that CS supplementa- tion increased rumen total volatile fatty acids (VFA) concentration and total-tract neutral detergent fibre (NDF) digestibility in bulls (Shang et al., 2020) or goats (Zhang et al., 2007), gas and short-chain fatty acids production in vitro (Vázquez-Armijo et al., 2011), and rumen cellulase activity and total bacteria and fungi populations in bulls (Shang et al., 2020). So, it can be suggested that dietary $\mathrm{Cu}$ was essential for both animal per se and ruminal microbes. LaBella et al. (1973) found that $\mathrm{Cu}$ addition stimulated 
growth hormone secretion of bovine pituitary in vitro. Growth hormone binds with its receptor, and then promotes protein synthesis and animal growth by stimulating the secretion of insulin-like growth factor-1 (IGF-1) (Breier, 1999). It has been reported that growth performance was improved by dietary CS supplementation in calves (Gengelbach and Spears, 1998) or bulls (Shang et al., 2020). Nevertheless, the impacts of dietary CS addition on IGF-1 secretion and expression of genes related to protein synthesis have not been examined in bulls.

Copper sulphate is a commonly used $\mathrm{Cu}$ supplement in ruminants (NRC, 2001). The free $\mathrm{Cu}^{2+}$ from CS could form insoluble complex with sulphur and molybdenum in the rumen, thereby the absorption rate of dietary $\mathrm{Cu}$ was only $5-10 \%$ in adult ruminants (NRC, 2001; Spears, 2003). It was proposed that rumen-protected $\mathrm{Cu}$ source should be used to reduce the amount of $\mathrm{Cu}$ excretion in ruminants (Ward et al., 1993; Engle and Spears, 2000b). However, the comparison between the effects of addition of CS and rumen-protected CS (RPCS) on performance, nutrient digestion and rumen fermentation in bulls has not been done yet.

Based on the information presented above, it was hypothesized that bulls receiving RPCS addition would have greater increase in weight gain than those consuming diets with CS addition. Therefore, the aim of the study was to evaluate the effects of CS and RPCS addition on performance, nutrient digestibility, rumen fermentation and hepatic gene expression in bulls.

\section{Material and methods}

\section{Supplements of CS and RPCS}

Copper sulphate $\left(\mathrm{CuSO}_{4} * 5 \mathrm{H}_{2} \mathrm{O}, 256 \mathrm{~g} / \mathrm{kg}\right.$ of $\left.\mathrm{Cu}\right)$ was purchased commercially, and RPCS $(80 \mathrm{~g} / \mathrm{kg}$ of $\mathrm{Cu}$ ) was manufactured according to the method of Wang et al. (2016). The RPCS contained $312.5 \mathrm{~g} / \mathrm{kg}$ of $\mathrm{CuSO}_{4} * 5 \mathrm{H}_{2} \mathrm{O}, 187.5 \mathrm{~g} / \mathrm{kg}$ of silicon dioxide, $340 \mathrm{~g} / \mathrm{kg}$ of hydrogenated fat (ratio of C16:0 and $\mathrm{C} 18: 0-2: 1)$ and $160 \mathrm{~g} / \mathrm{kg}$ of calcium stearate. Hydrogenated fat was heated to $80{ }^{\circ} \mathrm{C}$, and then blended with $\mathrm{CuSO}_{4} * 5 \mathrm{H}_{2} \mathrm{O}$, silicon dioxide and calcium stearate to form a mixture. The mixture was pelleted to $1-1.25 \mathrm{~cm}$ particles using a rotating pelletizer (HJ-400-S; Chongqing Rongkai Machinery Manufacturing Co. Ltd, Chongqing, China). The release ratio of CS from RPCS in the rumen and in the small intestine was determined by using four cannulated Simmental steers (546 $\pm 18.6 \mathrm{~kg}$ of body weight $(\mathrm{BW})$ ), and was 0.24 and 0.60 , respectively.
Briefly, 3 RPCS samples, 6 replicates and $5 \mathrm{~g}$ of each, were put into nylon bags $(12 \times 8 \mathrm{~cm}$; pore $37 \mu \mathrm{m})$, and then incubated in the rumen of each steer for $24 \mathrm{~h}$. Three replicates of each sample collected from the rumen were used to determine the release ratio of CS from RPCS in the intestine. Three nylon bags $(6 \times 3 \mathrm{~cm}$; pore $37 \mu \mathrm{m})$ with $1.5 \mathrm{~g}$ sample of each bag were put into the duodenum cannula of each steer, and were collected from the faeces. Bags collected from the rumen and from the faeces were washed in cool water for 3 min using a washer, and dried at $55^{\circ} \mathrm{C}$ for $48 \mathrm{~h}$ in a forced air oven. The contents of dry matter $(\mathrm{DM})$ and $\mathrm{Cu}$ were measured for each sample.

\section{Animals and experimental design}

The experiment followed the procedures of Animal Care and Use Committee of Shanxi Agriculture University. Thirty-six Holstein dairy bulls (not castrated) aging $15 \pm 0.9$ months and weighing $465 \pm 13.2 \mathrm{~kg} \mathrm{BW}$ were selected, and randomly assigned to three groups: control (without $\mathrm{Cu}$ addition), $\mathrm{CS}$ ( $8 \mathrm{mg} / \mathrm{kg} \mathrm{DM} \mathrm{Cu}$ as CS) or RPCS $(8 \mathrm{mg} / \mathrm{kg} \mathrm{DM} \mathrm{Cu}$ as RPCS). The study lasted for 80 days with 20 days for adaptation and 60 days for sample collection. Basal diet was formulated according to NRC (2001) to meet the nutrient requirements of $450 \mathrm{~kg} \mathrm{BW}$ dairy bulls (Table 1).

Table 1. Ingredient and chemical composition of the basal diets, $\mathrm{g} / \mathrm{kg}$ dry matter

\begin{tabular}{lc}
\hline Indices & Content \\
\hline Ingredient & \\
maize silage & 500 \\
maize grain, ground & 234 \\
wheat bran & 40 \\
soybean meal & 30 \\
rapeseed meal & 70 \\
cottonseed cake & 100 \\
calcium carbonate & 15 \\
salt & 5 \\
calcium phosphate & 5 \\
mineral and vitamin premix & 1 \\
Chemical composition & 1 \\
organic matter & \\
crude protein & 901 \\
ether extract & 160 \\
neutral detergent fibre & 25.3 \\
acid detergent fibre & 385 \\
non-fibre carbohydrate & 259 \\
calcium & 331 \\
phosphorus & 8.10 \\
sulphur & 4.70 \\
copper, mg/kg & 2.13 \\
molybdenum, mg/kg & 7.63 \\
\hline
\end{tabular}

${ }_{1}^{1}$ provided per kg of diet, mg: Fe $50, \mathrm{Mn} 35, \mathrm{Zn} 30,10.3$, Se 0.3, Co 0.1, IU: vit. A 7600, vit. D 1300, vit. E 45; ${ }^{2}$ calculated by 1000 - crude protein - neutral detergent fibre - fat - ash 
Supplementary CS and RPCS were mixed into concentrate before the trial. Copper content in diets was $7.63,15.6$ and $15.6 \mathrm{mg} / \mathrm{kg} \mathrm{DM}$ for control, CS and RPCS groups, respectively. Bulls were raised in individual pens $(3 \times 3 \mathrm{~m})$, had free access to drinking water and were fed at 7:00 and 19:00 each day ad libitum. Refusals did not exceed 5\%.

\section{Data and sample collection}

Bulls were weighed on days 1, 30 and 60 of the sample collection period. Feed offered and refused were measured daily to determine DM intake (DMI). Samples of feed offered and refused were collected once a week, and stored at $-20{ }^{\circ} \mathrm{C}$. During days $26-30$ and 56-60, all bulls had a harness system fitted with a faecal collection bag installed. Total excretion of faeces was weighed, and sampled (1/20 of wet faeces weight) daily for each bull. Faecal sample was blended with $100 \mathrm{~g} / 1$ tartaric acid solution based on one quarter of sample quality, and stored at $-20{ }^{\circ} \mathrm{C}$. At the end of the trial, samples of feed, refusals and faeces were dried at $55{ }^{\circ} \mathrm{C}$ for $48 \mathrm{~h}$, ground to pass through a $1-\mathrm{mm}$ screen, and then pooled by bull for chemical analyses.

Ruminal fluid of each bull was sampled at 10:00 on days 25 and 55 by using a stomach tube. To avoid saliva contamination, the first collected $200 \mathrm{ml}$ of ruminal fluid was discarded. Determination of ruminal fluid $\mathrm{pH}$ was performed using a portable $\mathrm{pH}$ meter (HK-1309pH, Beijing Huakeyi technology Co. LTD, Beijing, China), and then filtered through four layers of medical gauze. Filtrates used for measuring VFA $(5 \mathrm{ml})$ and ammonia- $\mathrm{N}(5 \mathrm{ml})$ were mixed with $1 \mathrm{ml}$ of $250 \mathrm{~g} / 1$ meta-phosphoric acid and $1 \mathrm{ml}$ of $20 \mathrm{~g} / 1$ sulphuric acid, respectively, and stored at $-20^{\circ} \mathrm{C}$. Filtrates used for DNA extraction $(5 \mathrm{ml})$ and microbial enzyme activity determination $(50 \mathrm{ml})$ were frozen at $-80{ }^{\circ} \mathrm{C}$.

On days 30 and 60 of the collection period, after local anaesthesia, liver samples for each bull were taken by blind percutaneous needle biopsy $(14 \mathrm{G} \times 152 \mathrm{~mm}$; Dispomed Witt oHG, Gelnhausen, Germany) (Gross et al., 2011). Liver sample was placed into a RNA stabilization reagent (RNAlater; Zhengzhou Baiji Biotechnology Co. LTD, Zhengzhou, China), kept at $4{ }^{\circ} \mathrm{C}$ for $24 \mathrm{~h}$, and then frozen at $-80{ }^{\circ} \mathrm{C}$ before analysis.

\section{Analytical methods}

According to AOAC International (2000), samples of diets, refusals and faeces were analysed for DM (method 934.01), crude ash (method 942.05), $\mathrm{N}$ (method 976.05) and acid detergent fibre (ADF) (method 973.18). Contentoforganic matter(OM) was calculated by the difference of DM and ash. Content of NDF was analysed based on the method of Van Soest et al. (1991) with heat stable $\alpha$-amylase and sodium sulphite used and expressed inclusive of residual ash. Contents of $\mathrm{Ca}, \mathrm{Mg}, \mathrm{P}, \mathrm{S}, \mathrm{Cu}, \mathrm{Fe}$, $\mathrm{Zn}, \mathrm{Mn}$ and $\mathrm{Mo}$ in diets and $\mathrm{Cu}$ in the liver were measured by an inductively coupled plasma optical emission spectrophotometer (ICP-OES, Optima 7300 DV, Perkin Elmer, Waltham, MA, USA) based on the procedure described by Webb et al. (2014). Rumen VFA were measured by gas chromatography (GC-8890B; Liaoning Dongke Analytical Instrument Co. LTD, China) with 2-ethylbutyric acid as internal standard, and ammonia-N was analysed using a colorimetric spectrophotometer (P8, Shanghai Meipuda Instrument Co., LTD, Shanghai, China) according to the method of AOAC International (2000). Microbial enzymatic activites of carboxymethyl-cellulase (CMC), $\alpha$-amylase, xylanase, protease (Agarwal et al., 2002), laccase (Rodrigues et al., 2008), cellobiase and pectinase (Miller, 1959) were determined and expressed as reducing sugars $(\mu \mathrm{mol} / \mathrm{min} / \mathrm{ml})$ produced under the assay conditions.

\section{Extraction of microbial DNA and real-time PCR}

The homogenized rumen fluid $(1.5 \mathrm{ml})$ was used for total microbial DNA isolation by using the repeated bead-beating plus column method (Yu and Morrison, 2004). The quality and quantity of microbial DNA was determined via agarose gel electrophoresis and a NanoDrop 2000 Spectrophotometer (Thermo Fisher Scientific, Waltham, MA, USA), respectively. Primer sets of target microbes were designed according to Zhou et al. (2012) and Denman and McSweeney (2006), and are shown in Table 2. The sample-derived standards of all target genes were prepared from the treatment pool set of microbial DNA. Sample-derived DNA standards for every qPCR assay were generated by using the regular PCR. Subsequently, the PCR product was purified using a MiniBEST DNA Fragment Purification Kit (Takara Biotechnology Co., Ltd., Beijing, China) and quantified by a NanoDrop 2000 Spectrophotometer (Thermo Fisher Scientific, Waltham, MA, USA). Copy number concentration of each samplederived standard was evaluated according to the PCR product length and its mass concentration, and the target DNA was quantified by using serial 10 -fold gradient dilutions from $10^{1}$ to $10^{8}$ DNA copies (Kongmun et al., 2010). Amplification and detection of qPCR were carried out in a StepOne ${ }^{\mathrm{TM}}$ system (Thermo Fisher Scientific, Waltham, MA, USA). Samples were assayed in triplicate. 
Table 2. PCR primers of microbes for real-time PCR assay

\begin{tabular}{|c|c|c|c|c|}
\hline Target species & Primer sequence $\left(5^{\prime}-3^{\prime}\right)$ & $\begin{array}{l}\text { GeneBank accession } \\
\text { no. }\end{array}$ & $\begin{array}{l}\text { Annealing } \\
\text { temperature, }{ }^{\circ} \mathrm{C}\end{array}$ & Size, bp \\
\hline Total bacteria & $\begin{array}{l}\text { F: CGGCAACGAGCGCAACCC } \\
\text { R: CCATTGTAGCACGTGTGTAGCC }\end{array}$ & СР058023.1 & 60 & 147 \\
\hline Total fungi & $\begin{array}{l}\text { F: GAGGAAGTAAAAGTCGTAACAAGGTTTC } \\
\text { R: CAAATTCACAAAGGGTAGGATGATT }\end{array}$ & GQ355327.1 & 57.5 & 120 \\
\hline Total protozoa & $\begin{array}{l}\text { F: GCTTTCGWTGGTAGTGTATT } \\
\text { R: CTTGCCCTCYAATCGTWCT }\end{array}$ & HM212038.1 & 59 & 234 \\
\hline Total methanogens & $\begin{array}{l}\text { F: TTCGGTGGATCDCARAGRGC } \\
\text { R: GBARGTCGWAWCCGTAGAATCC }\end{array}$ & GQ339873.1 & 60 & 160 \\
\hline Ruminococcus albus & $\begin{array}{l}\text { F: CCCTAAAAGCAGTCTTAGTTCG } \\
\text { R: CCTCCTTGCGGTTAGAACA }\end{array}$ & СР002403.1 & 60 & 176 \\
\hline Ruminococcus flavefaciens & $\begin{array}{l}\text { F: ATTGTCCCAGTTCAGATTGC } \\
\text { R: GGCGTCCTCATTGCTGTTAG }\end{array}$ & AB849343.1 & 60 & 173 \\
\hline Butyrivibrio fibrisolvens & $\begin{array}{l}\text { F: ACCGCATAAGCGCACGGA } \\
\text { R: CGGGTCCATCTTGTACCGATAAAT }\end{array}$ & HQ404372.1 & 61 & 65 \\
\hline Fibrobacter succinogenes & $\begin{array}{l}\text { F: GTTCGGAATTACTGGGCGTAAA } \\
\text { R: CGCCTGCCCCTGAACTATC }\end{array}$ & AB275512.1 & 61 & 121 \\
\hline Ruminobacter amylophilus & $\begin{array}{l}\text { F: CTGGGGAGCTGCCTGAATG } \\
\text { R: GCATCTGAATGCGACTGGTTG }\end{array}$ & MH708240.1 & 60 & 102 \\
\hline Prevotella ruminicola & $\begin{array}{l}\text { F: GAAAGTCGGATTAATGCTCTATGTTG } \\
\text { R: CATCCTATAGCGGTAAACCTTTGG }\end{array}$ & LT975683.1 & 58.5 & 74 \\
\hline
\end{tabular}

The reaction mixture of $20 \mu 1$ included $10 \mu 1$ SYBR Premix Ex Taq ${ }^{\mathrm{TM}} \mathrm{II}$ (TaKaRa Biotechnology Co., Ltd, Dalian, China), $2 \mu$ l template DNA, $0.8 \mu$ of each primer $(10 \mu \mathrm{M}), 0.4 \mu 1$ ROX Reference Dye II and $6.0 \mu \mathrm{l}$ double standard sterile water. The qPCR reaction conditions were: 1 cycle of $50^{\circ} \mathrm{C}$ for $2 \mathrm{~min}$ and $95^{\circ} \mathrm{C}$ for $2 \mathrm{~min}$ for initial denaturation, followed by 45 cycles of $95^{\circ} \mathrm{C}$ for $15 \mathrm{~s}$, at annealing temperature for $1 \mathrm{~min}$, and then product elongation at $72{ }^{\circ} \mathrm{C}$ for $30 \mathrm{~s}$. Specificity of amplification was performed via dissociation curve analysis of PCR end products by increasing the temperature at a rate of $1{ }^{\circ} \mathrm{C}$ every 30 s from $60{ }^{\circ} \mathrm{C}$ to $95^{\circ} \mathrm{C}$.

\section{Extraction of hepatic RNA and real-time PCR}

Total RNA was extracted from the liver biopsies using a Total RNA isolation kit (Invitrogen, Carlsbad, CA, USA). An iScriptTM cDNA Synthesis Kit (Bio-Rad Laboratories GmbH, Munich, Germany) was used to synthesize cDNA from $500 \mathrm{ng}$ total RNA according to the manufacturer's instructions. Reaction condition was: $15 \mathrm{~min}$ at $37^{\circ} \mathrm{C}$ and $5 \mathrm{~min}$ at $85{ }^{\circ} \mathrm{C}$. Negative control reaction without reverse transcriptase was performed on each sample to detect possible contamination of genomic DNA or environmental DNA. Primer sets of target genes were designed according to $\mathrm{Hu}$ et al. (2016), and are shown in Table 3. The target fragments of liver gene were amplified by regular PCR with each primer set sequences (Han et al., 2009). The qPCR assays for $I G F-1$, IGF-1 receptor $(I G F-1 R)$, phosphoinositide 3-kinase $(P I 3 K)$, mammalian target of rapamycin $(m T O R)$ and ribosomal protein S6 kinase (RPS6KB 1$)$ were performed using a StepOne ${ }^{\mathrm{TM}}$ system (Thermo Fisher Scientific, Waltham, MA, USA), as described in microbial DNA amplification and detection.

\section{Statistical analyses}

Measurements of DMI was reduced to monthly means before statistical analysis. Feed conversion ratio (FCR) of each bull was estimated as dry matter intake (DMI) divided by average daily gain (ADG). Each replicate served as an experimental unit. Oneway analysis of variance with the Tukey's multiple comparison post-test was performed to determine significant difference among the treatments in BW, DMI, ADG and FCR in two sampling periods (days 1-30 and 31-60). Data on DMI, ADG and FCR were further analysed as repeated measurements with a first-order autoregressive-covariance structure using a mixed model procedure of SAS (2002). The model contained the fixed effects of treatments, month, treatment by month interaction and the residual error. Rumen fermentation parameters, apparent nutrient digestibility, microbial enzymatic activity, microbial copy and hepatic parameters were also analysed as repeated measurements. 
Table 3. PCR primers of hepatic gene expression for real-time PCR assay

\begin{tabular}{lllll}
\hline Gene & Primer sequence & GenBank accession no. & $\begin{array}{l}\text { Annealing } \\
\text { temperature, }{ }^{\circ} \mathrm{C}\end{array}$ & Size, bp \\
\hline IGF-1 & $\begin{array}{l}\text { Fwd-5'-ATCAGCAGTCTTCCAACCCAAT -3' } \\
\text { Rev-5'- TGAAGGCGAGCAAGCACAG -3' }\end{array}$ & BC126802.1 & 60 & 121 \\
IGF-1R & $\begin{array}{l}\text { Fwd-5'- CGGCTCAACCCAGGGAACTA -3' } \\
\text { Rev-5'- CCACTATCAACAGAACCGCAATG -3' }\end{array}$ & NM_001244612.1 & 60 & 163 \\
PI3K & $\begin{array}{l}\text { Fwd-5'- GCCAAGCATTGTTGAAGGGT -3' } \\
\text { mev-5'- GCACCAGCCGATCTACAAAAG -3' }\end{array}$ & NM_001099067.1 & 60 & 87 \\
\multirow{2}{*}{ RPS6KB1 } & $\begin{array}{l}\text { Fwd-5'- CATCATTCGCATTCAGTCCATC -3' } \\
\text { Rev-5'- TCCTGCCGCAAGTCCTCAT -3' }\end{array}$ & XM_005901305.1 & 60 & 138 \\
& Rev-5'- CAAGCCTCCCCGCTCATCGTCA -3' & NM_205816.1 & 60 & 90 \\
\hline
\end{tabular}

IGF-1 - insulin-like growth factor-1, IGF-1R - insulin-like growth factor-1 receptor, PI3K - phosphoinositide 3-kinase, $m$ TOR - mammalian target of rapamycin, RPS6KB1 - ribosomal protein S6 kinase

Mean separations using probability of difference tests (PDIFF in SAS) were conducted only for treatment $\times$ time interaction that were statistically significant $(P<0.050)$. Significant differences were suggested at $P<0.050$.

\section{Results}

\section{Performance}

The treatment $\times$ time interaction was not significant for DMI, ADG and FCR (Table 4). Dietary CS or RPCS provisions did not affect DMI, ADG and FCR in dairy bulls.

\section{Apparent nutrients digestibility and rumen fermentation}

Significant treatment $\times$ time interaction was not found for nutrients digestibility and rumen fermentation parameters (Table 5). Apparent digestibilities of DM, OM, CP, NDF and ADF increased $(P<0.05)$ with CS or RPCS addition. Bulls receiving CS or RPCS addition had lower $(P=0.043)$ rumen $\mathrm{pH}$ and higher $(P=0.016)$ total VFA concentration in comparison with those in control. Acetate percentage and acetate to propionate ratio increased $(P<0.05)$, but propionate percentage and ammonia $\mathrm{N}$ concentration decreased $(P<0.05)$ with dietary

Table 4. Effects of copper sulphate (CS) and rumen-protected copper sulphate (RPCS) addition on dry matter (DM) intake, average daily gain (ADG) and feed conversion ratio (FCR) in dairy bulls

\begin{tabular}{|c|c|c|c|c|c|c|c|}
\hline \multirow{2}{*}{ Indices } & \multicolumn{3}{|c|}{ Treatment $^{1}$} & \multirow{2}{*}{ SEM } & \multicolumn{3}{|l|}{$P$-value } \\
\hline & control & CS & RPCS & & treatment & time & treatment $\times$ time \\
\hline \multicolumn{8}{|c|}{ DM intake, kg/day } \\
\hline days $1-30$ & 10.1 & 10.1 & 10.4 & 0.338 & 0.923 & & \\
\hline days $31-60$ & 10.2 & 10.5 & 10.7 & 0.306 & 0.804 & & \\
\hline overall & 10.1 & 10.3 & 10.6 & 0.258 & 0.781 & 0.571 & 0.938 \\
\hline \multicolumn{8}{|l|}{ Body weight, $\mathrm{kg}$} \\
\hline day 1 & 463 & 459 & 475 & 13.2 & 0.381 & & \\
\hline day 30 & 500 & 501 & 490 & 13.1 & 0.363 & & \\
\hline day 60 & 539 & 534 & 550 & 12.9 & 0.398 & & \\
\hline \multicolumn{8}{|l|}{ ADG, g/day } \\
\hline days $1-30$ & 1223 & 1383 & 1173 & 43 & 0.154 & & \\
\hline days $31-60$ & 1321 & 1112 & 1312 & 66 & 0.394 & & \\
\hline overall & 1274 & 1253 & 1241 & 39 & 0.928 & 0.915 & 0.098 \\
\hline \multicolumn{8}{|l|}{$\mathrm{FCR}, \mathrm{kg} \mathrm{DM} / \mathrm{kg}$} \\
\hline days $1-30$ & 11.1 & 9.30 & 12.0 & 0.548 & 0.178 & & \\
\hline days 31-60 & 10.1 & 14.0 & 10.9 & 0.908 & 0.220 & & \\
\hline overall & 10.6 & 11.6 & 11.5 & 0.502 & 0.644 & 0.406 & 0.059 \\
\hline
\end{tabular}

${ }^{1}$ control, CS and RPCS were supplemented with $0,8.0 \mathrm{mg} / \mathrm{kg} \mathrm{DM}$ of Cu from CS, and $8.0 \mathrm{mg} / \mathrm{kg}$ DM of Cu from RPCS, respectively; SEM - standard error of the mean; values are presented by mean $(n=12)$ 
Table 5. Effects of copper sulphate (CS) and rumen-protected copper sulphate (RPCS) addition on apparent total-tract nutrient digestibility and ruminal fermentation in dairy bulls

\begin{tabular}{|c|c|c|c|c|c|c|c|}
\hline \multirow{2}{*}{ Indices } & \multicolumn{3}{|c|}{ Treatment $^{1}$} & \multirow{2}{*}{ SEM } & \multicolumn{3}{|l|}{$P$-value } \\
\hline & control & CS & RPCS & & treatment & time & treatment $\times$ time \\
\hline \multicolumn{8}{|l|}{ Apparent digestibility, \% } \\
\hline dry matter (DM) & $67.9^{b}$ & $69.9^{\mathrm{a}}$ & $70.8^{\mathrm{a}}$ & 0.443 & 0.009 & 0.458 & 0.651 \\
\hline organic matter & $70.2^{b}$ & $73.7^{\mathrm{a}}$ & $72.2^{\mathrm{a}}$ & 0.507 & 0.005 & 0.341 & 0.726 \\
\hline crude protein & $68.7^{b}$ & $72.2^{\mathrm{a}}$ & $72.3^{\mathrm{a}}$ & 0.636 & 0.002 & 0.361 & 0.602 \\
\hline neutral detergent fibre & $60.2^{b}$ & $63.2^{\mathrm{a}}$ & $62.9^{a}$ & 0.478 & 0.004 & 0.389 & 0.745 \\
\hline acid detergent fibre & $50.4^{b}$ & $52.4^{\mathrm{a}}$ & $52.9^{\mathrm{a}}$ & 0.612 & 0.026 & 0.474 & 0.629 \\
\hline \multicolumn{8}{|c|}{ Ruminal fermentation, $\mathrm{mmol} / 100 \mathrm{mmol}$} \\
\hline acetate $(\mathrm{A})$ & $62.9^{b}$ & $64.3^{\mathrm{a}}$ & $64.9^{\mathrm{a}}$ & 0.424 & 0.034 & 0.104 & 0.526 \\
\hline propionate $(\mathrm{P})$ & $21.8^{\mathrm{a}}$ & $20.1^{b}$ & $20.0^{\mathrm{b}}$ & 0.175 & 0.042 & 0.161 & 0.643 \\
\hline butyrate & 11.6 & 11.6 & 11.1 & 0.156 & 0.165 & 0.211 & 0.826 \\
\hline valerate & 1.65 & 1.73 & 1.62 & 0.051 & 0.865 & 0.358 & 0.667 \\
\hline isobutyrate & 0.83 & 0.92 & 0.92 & 0.028 & 0.599 & 0.218 & 0.518 \\
\hline isovalerate & 1.19 & 1.35 & 1.41 & 0.061 & 0.622 & 0.397 & 0.776 \\
\hline$A: P$ & $2.88^{b}$ & $3.19^{a}$ & $3.25^{\mathrm{a}}$ & 0.026 & 0.012 & 0.259 & 0.657 \\
\hline Ammonia N, mg/100 ml & $14.6^{\mathrm{a}}$ & $13.0^{\mathrm{b}}$ & $13.1^{b}$ & 0.275 & 0.010 & 0.635 & 0.589 \\
\hline $\mathrm{pH}$ & $6.98^{a}$ & $6.68^{b}$ & $6.72^{b}$ & 0.025 & 0.043 & 0.167 & 0.591 \\
\hline Total VFA, mM & $93.77^{\mathrm{b}}$ & $104.4^{\mathrm{a}}$ & $106.5^{a}$ & 1.584 & 0.016 & 0.352 & 0.448 \\
\hline
\end{tabular}

${ }_{1}^{1}$ control, CS and RPCS were supplemented with $0,8.0 \mathrm{mg} / \mathrm{kg}$ DM of Cu from CS, and $8.0 \mathrm{mg} / \mathrm{kg} \mathrm{DM} \mathrm{of} \mathrm{Cu} \mathrm{from} \mathrm{RPCS,} \mathrm{respectively;}$ SEM - standard error of the mean; VFA - volatile fatty acids; abc - means with different superscripts in each row are significantly different at $P<0.05$; values are presented by mean $(n=12)$

Table 6. Effects of copper sulphate (CS) and rumen-protected copper sulphate (RPCS) addition on ruminal microbial enzyme activity and microbiota in dairy bulls

\begin{tabular}{|c|c|c|c|c|c|c|c|}
\hline \multirow{2}{*}{ Indices } & \multicolumn{3}{|c|}{ Treatment $^{1}$} & \multirow{2}{*}{ SEM } & \multicolumn{3}{|l|}{$P$-value } \\
\hline & control & CS & RPCS & & treatment & time & treatment $\times$ time \\
\hline \multicolumn{8}{|l|}{ Microbial enzyme activity² } \\
\hline carboxymethyl-cellulase & $0.228^{b}$ & $0.242^{\mathrm{a}}$ & $0.242^{\mathrm{a}}$ & 0.003 & 0.008 & 0.565 & 0.664 \\
\hline cellobiase & 0.122 & 0.065 & 0.079 & 0.011 & 0.095 & 0.324 & 0.539 \\
\hline xylanase & $0.764^{c}$ & $0.826^{b}$ & $0.904^{\mathrm{a}}$ & 0.017 & 0.004 & 0.474 & 0.615 \\
\hline pectinase & $0.375^{b}$ & $0.384^{b}$ & $0.401^{\mathrm{a}}$ & 0.005 & 0.005 & 0.352 & 0.758 \\
\hline laccase & $3.38^{c}$ & $4.65^{\mathrm{a}}$ & $4.02^{b}$ & 0.161 & 0.001 & 0.487 & 0.642 \\
\hline a-amylase & $0.670^{\mathrm{a}}$ & $0.451^{c}$ & $0.585^{b}$ & 0.029 & 0.009 & 0.497 & 0.526 \\
\hline protease & $0.346^{b}$ & $0.439^{a}$ & $0.348^{b}$ & 0.013 & 0.037 & 0.308 & 0.604 \\
\hline \multicolumn{8}{|l|}{ Microbiota, copies/ml } \\
\hline total bacteria, $\times 10^{11}$ & $1.22^{b}$ & $1.75^{\mathrm{a}}$ & $1.73^{\mathrm{a}}$ & 0.073 & 0.039 & 0.226 & 0.543 \\
\hline total anaerobic fungi, $\times 10^{9}$ & 2.58 & 2.76 & 3.08 & 0.116 & 0.217 & 0.453 & 0.539 \\
\hline total protozoa, $\times 10^{7}$ & 1.95 & 2.00 & 2.37 & 0.091 & 0.117 & 0.374 & 0.653 \\
\hline total methanogens, $\times 10^{9}$ & $7.99^{a}$ & $6.50^{\mathrm{b}}$ & $7.80^{\mathrm{ab}}$ & 0.261 & 0.023 & 0.224 & 0.834 \\
\hline Ruminococcus albus, $\times 10^{7}$ & $8.88^{b}$ & $9.68^{\mathrm{a}}$ & $9.74^{\mathrm{a}}$ & 0.627 & 0.021 & 0.371 & 0.689 \\
\hline Ruminococcus flavefaciens, $\times 10^{8}$ & $0.64^{b}$ & $0.53^{b}$ & $1.10^{\mathrm{a}}$ & 0.076 & 0.009 & 0.231 & 0.523 \\
\hline Fibrobacter succinogenes, $\times 10^{10}$ & 1.78 & 1.76 & 1.84 & 0.063 & 0.892 & 0.441 & 0.789 \\
\hline Butyrivibrio fibrisolvens, $\times 10^{9}$ & $4.15^{c}$ & $5.20^{\mathrm{b}}$ & $6.02^{\mathrm{a}}$ & 0.249 & 0.003 & 0.222 & 0.671 \\
\hline Prevotella ruminicola, $\times 10^{10}$ & 3.12 & 3.78 & 3.82 & 0.198 & 0.271 & 0.365 & 0.461 \\
\hline Ruminobacter amylophilus, $\times 10^{8}$ & $2.47^{\mathrm{b}}$ & $2.85^{\mathrm{ab}}$ & $3.13^{\mathrm{a}}$ & 0.101 & 0.002 & 0.387 & 0.607 \\
\hline
\end{tabular}

${ }^{1}$ control, CS and RPCS were supplemented with $0,8.0 \mathrm{mg} / \mathrm{kg}$ dry matter (DM) of Cu from CS, and $8.0 \mathrm{mg} / \mathrm{kg}$ DM of Cu from RPCS, respectively;

${ }^{2}$ units of enzyme activity are: carboxymethyl-cellulase ( $\mu \mathrm{mol}$ glucose $\left./ \mathrm{min} / \mathrm{ml}\right)$, cellobiase ( $\mu \mathrm{mol}$ glucose $\left./ \mathrm{min} / \mathrm{ml}\right)$, xylanase $(\mu \mathrm{mol}$ xylose $/ \mathrm{min} / \mathrm{ml})$, pectinase $(\mu \mathrm{mol} \mathrm{D}$-galactouronic acid $/ \mathrm{min} / \mathrm{ml})$, laccase $(\mathrm{U} / \mathrm{ml})$, a-amylase $(\mu \mathrm{mol}$ maltose $/ \mathrm{min} / \mathrm{ml})$ and protease $(\mu \mathrm{g}$ hydrolysed $\mathrm{protein} / \mathrm{min} / \mathrm{ml})$; SEM - standard error of the mean; abc - means with different superscripts in each row are significantly different at $P<0.05$; values are presented by mean $(n=12)$ 
Table 7. Effects of copper sulphate (CS) and rumen-protected copper sulphate (RPCS) addition on liver copper concentration and mRNA expression of gene related to protein synthesis in dairy bulls

\begin{tabular}{|c|c|c|c|c|c|c|c|}
\hline \multirow{2}{*}{ Indices } & \multicolumn{3}{|c|}{ Treatments $^{1}$} & \multirow{2}{*}{ SEM } & \multicolumn{3}{|l|}{$P$-value } \\
\hline & control & CS & RPCS & & treatment & time & treatment $\times$ time \\
\hline Liver Cu, mg/kg dry matter & $140^{c}$ & $161^{\mathrm{b}}$ & $178^{a}$ & 9.65 & 0.019 & 0.352 & 0.536 \\
\hline \multicolumn{8}{|l|}{ Gene expression², copies/mg } \\
\hline IGF-1, ×1011 & $10.3^{\mathrm{a}}$ & $9.81^{\mathrm{a}}$ & $8.29^{b}$ & 0.354 & 0.011 & 0.486 & 0.617 \\
\hline IGF-1R, $\times 10^{10}$ & $1.86^{a}$ & $1.76^{\mathrm{a}}$ & $1.45^{\mathrm{b}}$ & 0.063 & 0.035 & 0.316 & 0.575 \\
\hline$P I 3 K, \times 10^{10}$ & $3.40^{\mathrm{a}}$ & $3.18^{\mathrm{a}}$ & $2.69^{b}$ & 0.123 & 0.034 & 0.532 & 0.812 \\
\hline$m T O R, \times 10^{9}$ & $8.60^{\mathrm{a}}$ & $7.14^{b}$ & $6.18^{c}$ & 0.253 & 0.001 & 0.467 & 0.634 \\
\hline RPS6KB1, $\times 10^{10}$ & $6.81^{\mathrm{a}}$ & $6.48^{\mathrm{a}}$ & $5.62^{b}$ & 0.172 & 0.002 & 0.298 & 0.384 \\
\hline
\end{tabular}

${ }^{1}$ control, CS and RPCS were supplemented with $0,8.0 \mathrm{mg} / \mathrm{kg}$ dry matter (DM) of Cu from CS, and $8.0 \mathrm{mg} / \mathrm{kg}$ DM of Cu from RPCS, respectively; ${ }^{2}$ IGF-1 - insulin-like growth factor-1, IGF-1R - insulin-like growth factor-1 receptor, PI3K - phosphoinositide 3-kinase, $m$ TOR - mammalian target of rapamycin, RPS6KB1 - ribosomal protein S6 kinase; SEM - standard error of the mean; abc - means with different superscripts in each row are significantly different at $P<0.05$; values are presented by mean $(n=12)$

CS or PRCS provision. Molar percentages of butyrate, valerate, isovalerate and isobutyrate were not affected by dietary $\mathrm{Cu}$ addition. No significant difference was observed for nutrients digestibility and ruminal fermentation parameters between CS and RPCS supplementation.

\section{Rumen enzymatic activity and microbiota}

The treatment $\times$ time interaction was not significant for enzymatic activity and microbiota (Table 6). Activities of xylanase, carboxymethylcellulase and laccase increased $(P<0.05)$, $\alpha$-amylase decreased $(P=0.009)$, but cellobiase was unchanged with dietary CS or RPCS addition. Activities of $\alpha$-amylase and xylanase were higher $(P<0.05)$, but laccase was lower $(P=0.001)$ in animals fed RPCS than in those fed CS addition. Activity of pectinase was higher $(P=0.005)$ in RPCS group than in control and CS ones. Bulls receiving diet with $\mathrm{CS}$ addition had higher $(P=0.037)$ protease activity in comparison with those fed control and with RPCS addition diets. Populations of total bacteria, Ruminococcus albus and $B$. fibrisolvens increased $(P<0.05)$, but fungi, protozoa, Fibrobacter succinogenes and Prevotella ruminicola were unchanged by the addition of CS or RPCS to the diet. Population of methanogens was lower $(P=0.023)$ in CS group than in control and RPCS ones. Bulls consuming diets with RPCS addition had higher $(P<0.05)$ population of $B$. fibrisolvens in comparison with those receiving CS addition. Populations of $R$. flavefaciens and Ruminobacter amylophilus were not affected by CS addition, but increased $(P<0.05)$ with RPCS addition.

\section{Liver $\mathrm{Cu}$ concentration and gene expression}

The treatment $\times$ time interaction was not significant for hepatic $\mathrm{Cu}$ content and gene expression (Table 7). Liver $\mathrm{Cu}$ concentration was the highest for RPCS, followed by CS, and then control group. Hepatic mRNA expressions of $I G F-1, I G F-1 R$, RPS6KB1 and $P I 3 K$ were lower $(P<0.05)$ in RPCS group than in CS and control ones. Expression of $m T O R$ was the lowest for RPCS, followed by CS, and then control group.

\section{Discussion}

The unchanged ADG was likely associated with the limited response of DMI, indicating that $7.63 \mathrm{mg} / \mathrm{kg} \mathrm{DM}$ of $\mathrm{Cu}$ in the basal diets could meet the growth requirement of dairy bulls. Mills (1987) reported that hepatic $\mathrm{Cu}$ content usually ranges $100-150 \mathrm{mg} / \mathrm{kg}$ DM in growing steers with adequate $\mathrm{Cu}$ status. Bulls consuming control diets had hepatic $\mathrm{Cu}$ content of $140 \mathrm{mg} / \mathrm{kg} \mathrm{DM}$, and should be in adequate $\mathrm{Cu}$ status. Similarly, Ward et al. (1993) observed that addition of $\mathrm{Cu}$ in steers with adequate $\mathrm{Cu}$ status did not improve performance. Engle and Spears (2000b) found that feed intake, growth performance and feed efficiency of growing steers were unchanged after supplementing $20 \mathrm{mg} / \mathrm{kg} \mathrm{DM}$ $\mathrm{Cu}$ as $\mathrm{CS}$ in diets containing $10.2 \mathrm{mg} / \mathrm{kg} \mathrm{DM} \mathrm{Cu}$. Ruminal pH ranged from 6.98 to 6.68 , and was optimum for cellulolytic microbes growth and nutrients digestion (Russell and Wilson, 1996). The change of ruminal $\mathrm{pH}$ was associated with the increase of total VFA concentration with CS or RPCS addition. Similarly, Hasman et al. (2009) observed decreased media $\mathrm{pH}$ with $\mathrm{CS}$ addition 
in vitro. Dietary CS or RPCS inclusion changed the rumen fermentation mode to more acetate formation, as shown by the increase of acetate to propionate ratio. The changes of rumen total VFA concentration and acetate percentage were probably associated with the increase in activities of carboxymethylcellulase, xylanase and laccase and populations of total bacteria, $R$. albus and B. fibrisolvens. The results were in accordance with the increment in apparent digestibilities of DM, OM, NDF and ADF, indicating that dietary $\mathrm{Cu}$ provision was necessary for rumen cellulolytic microbial growth and fibre digestion. Shang et al. (2020) reported that rumen total bacteria and fungi population increased with supplementing $7.68 \mathrm{mg} / \mathrm{kg} \mathrm{DM} \mathrm{Cu}$ as $\mathrm{CS}$ in diets containing $8.72 \mathrm{mg} / \mathrm{kg} \mathrm{DM} \mathrm{Cu}$ of bulls. Copper, as a structural element or cofactor of redox enzyme, is essential for microbes (Ridge et al., 2008; Osman and Cavet, 2008), and is involved in the synthesis and activity of laccase (Palmieri et al., 2000). The cell walls of microbes and feed are negatively charged, thereby $\mathrm{Cu}^{2+}$ from $\mathrm{CS}$ could act as a bridge between feed and microbial cell (Lopez-Guisa and Satter, 1992). Similarly, Zhang et al. (2007) observed increased ruminal total VFA concentration and apparent fibre digestibility after supplementing $10 \mathrm{mg} / \mathrm{kg} \mathrm{DM} \mathrm{Cu}$ as CS in goat diets including $7.38 \mathrm{mg} / \mathrm{kg}$ DM Cu. Vázquez-Armijo et al. (2011) reported increased gas and short-chain fatty acids production after $21.7 \mathrm{mg} / \mathrm{kg} \mathrm{DM} \mathrm{Cu}$ as CS addition in vitro. However, in other studies it was found that cellulose digestion decreased with addition of $4 \mathrm{mg} / \mathrm{kg} \mathrm{DM} \mathrm{Cu}$ as CS in the cellulose substrate in vitro (Ward and Spears, 1993), and that rumen VFA molar proportion was unaffected by supplementing $10 \mathrm{mg} / \mathrm{kg} \mathrm{DM} \mathrm{Cu}$ as $\mathrm{CS}$ in a high-concentrate diets of steers (Engle and Spears, 2000a). Free $\mathrm{Cu}^{2+}$ from CS could form insoluble compounds with feed protein and carbohydrate in the rumen, causing the soluble $\mathrm{Cu}^{2+}$ less accessible for microbes (Hasman et al., 2009). Therefore, the divergent responses of rumen fermentation and nutrients digestibility to $\mathrm{Cu}$ addition were related to the difference of dietary composition in these studies. The propionate molar proportion decreasing with $\mathrm{Cu}$ addition was consistent with the observed reduction in $\alpha$-amylase activity. However, the change of ruminal ammonia- $\mathrm{N}$ concentration was not in accordance with the responses of protease activity and $R b$. amylophilus and $B$. fibrisolvens populations. Ruminal microbes use ammonia-N which mainly derived from feed protein degradation and VFA to synthesize protein (Pathak, 2008).
In view of the increase of total VFA concentration, the decrease of ammonia- $\mathrm{N}$ might be due to an enhancement in protein synthesis of microbes, as evidenced in the study of Shang et al. (2020). The increased hepatic $\mathrm{Cu}$ concentration indicated that the supplemented $\mathrm{Cu}$ was absorbed effectively. Such a finding was consistent with the results of Engle and Spears (2000b), where $\mathrm{Cu}$ concentration of the liver increased in steers receiving CS addition. The IGF-1 binds with IGF-1R, and then upregulates expressions of $P I 3 K, \quad m T O R$ and RPS6KB1, resulting in an increase in cell growth and protein synthesis (Pivonello et al., 2016). The changes of hepatic expressions of $m T O R, P I 3 K$ and $R P S 6 K B 1$ were consistent with that of $I G F-1$ and $I G F-1 R$, and might be a reason of the unchanged ADG with CS or RPCS addition.

When comparing $\mathrm{Cu}$ source at the same inclusion level $(8 \mathrm{mg} / \mathrm{kg} \mathrm{DM} \mathrm{Cu})$, no differences were observed in performance, apparent nutrients digestibility and rumen fermentation parameters between CS and RPCS addition. However, bulls consuming diets with RPCS addition had higher rumen activities of xylanase, pectinase and $\alpha$-amylase and populations of $R$. flavefaciens and B. fibrisolvens than those fed diets with CS addition. The results indicated that higher level of free $\mathrm{Cu}$ was not required for rumen microbial growth and enzyme activity. The release ratio of $\mathrm{Cu}^{2+}$ in the rumen of animals from CS and RPCS groups was 100 and 24\%, respectively. Osman and Cavet (2008) reported that high level of free $\mathrm{Cu}^{2+}$ in the rumen was toxic for microbes, since it could produce hydroxyl radicals, and displace native metal ions of protein and nucleic acids in microbial cells. When comparing with CS addition, higher $\mathrm{Cu}$ content and lower expressions of $I G F-1$, $I G F-1 R, m T O R, P I 3 K$ and RPS6KB1 in the liver were observed for RPCS addition. It can be suggested that more $\mathrm{Cu}$ was absorbed after the addition of RPCS than after CS one, and this had negative impacts on hepatic genes expression of protein synthesis metabolism. Therefore, the dietary addition level of $\mathrm{Cu}$ should be reduced when replacing $\mathrm{CS}$ with RPCS in dairy bulls.

\section{Conclusions}

The addition of $8 \mathrm{mg} / \mathrm{kg}$ dry matter of $\mathrm{Cu}$ as copper sulphate (CS) or rumen-protected copper sulphate (RPCS) in diets containing $7.63 \mathrm{mg} / \mathrm{kg}$ of $\mathrm{Cu}$ did not affect growth performance of bulls. Dietary $\mathrm{Cu}$ provision stimulated nutrients digestion, rumen fermentation and microbial growth. 
However, the dietary addition level of $\mathrm{Cu}$ should be reduced when replacing CS with RPCS in dairy bulls.

\section{Acknowledgements}

This work was supported by an Excellent Postgraduate Innovation project programme of Shanxi Province (2019SY212) and Animal Husbandry Dominant Key Discipline construction project in '1331 project' Key Discipline Construction plan of Shanxi Province. The authors thank the staff of Shanxi Agriculture University from the beef unit for care of the animals.

\section{References}

Agarwal N., Kamra D.N., Chaudhary L.C., Agarwal I., Sahoo A., Pathak N.N., 2002. Microbial status and rumen enzyme profile of crossbred calves fed on different microbial feed additives. Lett. Appl. Microbiol. 34, 329-336, https://doi. org/10.1046/j.1472-765x.2002.01092.x

AOAC International, 2000. Official Methods of Analysis of AOAC International. 17th Edition. Arlington, VA (USA)

Breier B.H., 1999. Regulation of protein and energy metabolism by the somatotropic axis. Domest. Anim. Endocrin. 17, 209-218, https://doi.org/10.1016/S0739-7240(99)00038-7

Denman S.E., McSweeney C.S., 2006. Development of a realtime PCR assay for monitoring anaerobic fungal and cellulolytic bacterial populations within the rumen. FEMS Microbiol. Ecol. 58, 572-582, https://doi.org/10.1111/j.15746941.2006.00190.x

Engle T.E., Spears J.W., 2000a. Dietary copper effects on lipid metabolism, performance, and ruminal fermentation in finishing steers. J. Anim. Sci. 78, 2452-2458, https://doi. org/10.2527/2000.7892452x

Engle T.E., Spears J.W., 2000b. Effects of dietary copper concentration and source on performance and copper status of growing and finishing steers. J. Anim. Sci. 78, 2446-2451, https:// doi.org/10.2527/2000.7892446x

Gengelbach G.P., Spears J.W., 1998. Effects of dietary copper and molybdenum on copper status, cytokine production, and humoral immune response of calves. J. Dairy Sci. 81, 32863292, https://doi.org/10.3168/jds.S0022-0302(98)75893-X

Gross J., Dorland H.A., Schwarz F.J., Bruckmaier R.M., 2011. Endocrine changes and liver mRNA abundance of somatotropic axis and insulin system constituents during negative energy balance at different stages of lactation in dairy cows. J. Dairy Sci. 94, 3484-3494, https://doi. org/10.3168/jds.2011-4251

Han H., Archibeque S.L., Engle T.E., 2009. Characterization and identification of hepatic mRNA related to copper metabolism and homeostasis in cattle. Biol. Trace Elem. Res. 129, 130-136, https://doi.org/10.1007/s12011-0088293-6

Hasman H., Bjerrum M.J., Christiansen L.E., Hansen H.C.B., Aarestrup F.M., 2009. The effect of pH and storage on copper speciation and bacterial growth in complex growth media. J. Microbiol. Methods 78, 20-24, https://doi.org/10.1016/j. mimet.2009.03.008
Hu R., Wang Z., Peng Q. et al., 2016. Effects of GHRP-2 and cysteamine administration on growth performance, somatotropic axis hormone and muscle protein deposition in yaks (Bos grunniens) with growth retardation. PLOS ONE 11, e0149461, https://doi.org/10.1371/journal.pone.0149461

Kongmun P., Wanapat M., Pakdee P., Navanukraw C., 2010. Effect of coconut oil and garlic powder on in vitro fermentation using gas production technique. Livest. Sci. 127, 38-44, https://doi. org/10.1016/j.livsci.2009.08.008

LaBella F., Dular R., Vivian S., Qeen G., 1973. Pituitary hormone releasing or inhibiting activity of metal ions present in hypothalamic extracts. Biochem. Biophys. Res. Commun. 52, 786-791, https://doi.org/10.1016/0006-291X(73)91006-1

Lopez-Guisa J.M., Satter L.D., 1992. Effect of copper and cobalt addition on digestion and growth in Heifers fed diets containing alfalfa silage or corn crop residues. J. Dairy Sci. 75, 247-256, https://doi.org/10.3168/jds.S0022-0302(92)77759-5

Miller G.L., 1959. Use of dinitrosalisylic acid reagent for determination of reducing sugar. Anal. Chem. 31, 426-428, https://doi. org/10.1021/ac60147a030

Mills C.F., 1987. Biochemical and physiological indicators of mineral status in animals: copper, cobalt and zinc. J. Anim. Sci. 65, 1702-1711, https://doi.org/10.2527/jas1987.6561702x

NRC, 2001. Nutrient Requirements of Dairy Cattle. 7th Revised Edition. National Academy Press. Washington, DC (USA)

Osman D., Cavet J.S., 2008. Copper homeostasis in bacteria. Adv. Appl. Microbiol. 65, 217-247, https://doi.org/10.1016/S00652164(08)00608-4

Palmieri G., Giardina P., Bianco C., Fontanella B., Sannia G., 2000. Copper induction of laccase isoenzymes in the ligninolytic fungus Pleurotus ostreatus. Appl. Environ. Microbiol. 66, 920-924, https://doi.org/10.1128/AEM.66.3.920-924.2000

PathakA.K., 2008. Various factors affecting microbial protein synthesis in the rumen. Vet. World 1, 186-189

Pivonello C., Negri M., De Martino M.C. et al., 2016. The dual targeting of insulin and insulin-like growth factor 1 receptor enhances the mTOR inhibitor-mediated antitumor efficacy in hepatocellular carcinoma. Oncotarget 7, 9718-9731, https:// doi.org/10.18632/oncotarget.6836

Ridge P.G., Zhang Y., Gladyshev V.N., 2008. Comparative genomic analyses of copper transporters and cuproproteomes reveal evolutionary dynamics of copper utilization and its link to oxygen. PLoS ONE 3, 1-9, https://doi.org/10.1371/journal. pone. 0001378

Rodrigues M.A., Pinto P.A., Bezerra R.M., Dias A.A., Guedes C.V.M., Cardoso V.M.G., Cone J.W., Ferreira L.M.M., Colaco J., Sequeir C.A., 2008. Effect of enzyme extracts isolated from white-rot fungi on chemical composition and in vitro digestibility of wheat straw. Anim. Feed Sci. Technol. 141, 326-338, https://doi.org/10.1016/j.anifeedsci.2007.06.015

Russell J.B., Wilson D.B., 1996. Why are ruminal cellulolytic bacteria unable to digest cellulose at low pH? J. Dairy Sci. 79, 15031509, https://doi.org/10.3168/jds.S0022-0302(96)76510-4

SAS, 2002. User's Guide: Statistics, Version 9 Edition. Statistical Analysis Systems Institute, Cary, NC (USA)

Shang X.K., Wang C., Zhang G.W., Liu Q., Guo G., Huo W.J., Zhang J., Pei C.X., 2020. Effects of soybean oil and dietary copper levels on nutrient digestion, ruminal fermentation, enzyme activity, microflora and microbial protein synthesis in dairy bulls. Arch. Anim. Nutr. 74, 257-270, https://doi.org/10.1080/ 1745039X.2019.1679562 
Spears J.W., 2003. Trace mineral bioavailability in ruminants. J. Nutr. 133, 1506-1509, https://doi.org/10.1093/jn/133.5.1506S

Van Soest P.J., Robertson J.B., Lewis B.A., 1991. Methods for dietary fiber, neutral detergent fiber and non-starch polysaccharides in relation to animal nutrition. J. Dairy Sci. 74, 3583-3597, https://doi.org/10.3168/jds.S0022-0302(91)78551-2

Vázquez-Armijo J.F., Martínez-Tinajero J.J., López D., Salem A.F.Z.M., Rojo R., 2011. In vitro gas production and dry matter degradability of diets consumed by goats with or without copper and zinc supplementation. Biol. Trace Elem. Res. 144, 580-587, https://doi.org/10.1007/s12011-011-9113-y

Wang C., Liu Q., Guo G., Huo W.J., Ma L., Zhang Y.L., Pei C.X., Zhang S.L., Wang H., 2016. Effects of rumen-protected folic acid on ruminal fermentation, microbial enzyme activity, cellulolytic bacteria and urinary excretion of purine derivatives in growing beef steers. Anim. Feed Sci. Technol. 221, 185-194, https:// doi.org/10.1080/1745039X.2016.1233677

Ward J.D., Spears J.W., 1993. Comparison of copper lysine and copper sulfate as copper sources for ruminants using in vitro methods. J. Dairy Sci. 76, 2994-2998, https://doi.org/10.3168/jds. S0022-0302(93)77638-9

Ward J.D., Spears J.W., Kegley E.B., 1993. Effect of copper level and source (copper lysine vs copper sulfate) on copper status, performance, and immune response in growing steers fed diets with or without supplemental molybdenum and sulfur. J. Anim. Sci. 71, 2748-2755, https://doi.org/10.2527/1993.71102748x
Webb S., Bartos J., Boles R., Hasty E., Thuotte E., Thiex N.J., 2014. Simultaneous determination of arsenic, cadmium, calcium, chromium, cobalt, copper, iron, lead, magnesium, manganese, molybdenum, nickel, selenium, and zinc in fertilizers by microwave acid digestion and inductively coupled plasmaoptical emission spectrometry detection: Single laboratory validation of a modification and extension of AOAC 2006.03. J. AOAC Int. 97, 700-711, https://doi.org/10.5740/ jaoacint.13-408

Yu Z., Morrison M., 2004. Improved extraction of PCR-quality community DNA from digesta and fecal sample. BioTechniques 36 , 808-812, https://doi.org/10.2144/04365ST04

Zhang W., Wang R.L., Zhu X.P., Kleemann D.O., Yue C.W., Jia Z.H., 2007. Effects of dietary copper on ruminal fermentation, nutrient digestibility and fibre characteristics in cashmere goats. Asian. Aust. J. Anim. Sci. 20, 1843-1848, https://doi. org/10.5713/ajas.2007.1843

Zhou Y.W., McSweeney C.S., Wang J.K., Liu J.X., 2012. Effects of disodium fumarate on ruminal fermentation and microbial communities in sheep fed on high-forage diets. Animal 6 , 815-823, https://doi.org/10.1017/S1751731111002102 